\title{
Series-Series Compensated Wireless Power Transfer at Two Different Resonant Frequencies
}

\author{
Shin-Young Cho, Il-Oun Lee, SangCheol Moon \\ Gun-Woo Moon \\ Department of Electrical Engineering \\ KAIST \\ Daejeon, Republic of Korea
}

\author{
Bong-Chul Kim and Ki Young Kim \\ Future IT Research Center \\ Samsung Advanced Institute of Technology \\ Yongin, Republic of Korea
}

\begin{abstract}
Recently, resonance phenomenon in wireless power transfer (WPT) is widely used to deliver power to a load over relatively large air gap via magnetic coupling. Since many reactive components in WPT system are contributed to the resonance phenomenon, there are many resonant frequencies. Theses resonant frequencies have different and distinguishing features. For this reason, the analysis of the resonant tank at various resonant frequencies is required to understand WPT system more easily. Therefore, in this paper, three resonant frequencies in series-series compensated WPT (SS-WPT) system are defined. And then, this paper is aiming to present the features of SS-WPT system in the major two of the three resonant frequencies. The analysis is supported with experimental results.
\end{abstract}

Keywords-Coupled energy transfer, inductive power transfer, series-series compensation, resonant converter, wireless power transfer.

\section{INTODUCTION}

When windings share a magnetic core and are close to each other, magnetic coupling is good and tight. This tightly magnetic coupling enables most of magnetic field generated by the one winding to pass through the other winding. For this reason, tightly magnetic coupling was the fundamental principle of transferring power effectively. On the contrary, when windings do not share a magnetic core and are instead separated over large air gap, magnetic coupling is bad and loose. As a result, most of magnetic field generated on one winding does not link with the other winding and return to the generated winding. Therefore, loosely magnetic coupling was considered as non-effective way to transfer power. However, loosely magnetic coupling has huge advantages of low maintenance cost, high reliability and the ability to operate in extreme environment from the absence of conductive contact. Furthermore, recently, resonance phenomenon is widely used to compensate the returned magnetic field in wireless power transfer (WPT) system [1]-[18] and thus begins to create new possibilities of effective power transmission.

Generally, capacitors are inserted to resonate with windings and overcome the returned magnetic field and low efficiency in WPT system. It is called as compensation. Since many reactive components are contributed to compensation, there are many resonant frequencies in compensated WPT system. Unlike the other frequencies, the resonant frequencies have different and distinguishing features. For this reason, the analysis of the resonant tank at various resonant frequencies is required to understand WPT system more easily.

Furthermore, in various research areas, the preferred resonant frequency is divided into two: one resonant frequency by self-inductance and resonant capacitor [1]-[18] and the other resonant frequency by leakage inductance and resonant capacitor [19], [20]. Therefore, the difference between two resonant frequencies is needed to be clarified.

In this paper, series-series compensated WPT (SS-WPT) system is analyzed at the major two resonant frequencies. First of all, the configuration and equivalent circuit of the SS-WPT system are explained and resonant frequencies are defined from the well-known open-circuit and short-circuit tests. And then, the features of the SS-WPT in the major two of the three resonant frequencies are presented. To analyze the SS-WPT system effectively, input impedance analysis and frequency domain analysis based on Fundamental Harmonic Approximation (FHA) are used. And the result of analysis is verified by experiment.

\section{STRUCTURE OF SS-WPT SYSTEM}

SS-WPT system consists of an input voltage source, a fullbridge inverter, a resonant tank, a full-bridge rectifier, a capacitive filter, and a load, as shown in Fig. 1. The full-bridge inverter connected to the input voltage source $V_{I N}$ applies a square wave of voltage to the resonant tank. The square wave of voltage $V_{A B}(t)$ can be described by

$$
\begin{aligned}
V_{A B}(t)=V_{I N} & (0<\omega t \leq \pi) \\
-V_{I N} & (\pi<\omega t \leq 2 \pi)
\end{aligned}
$$

As the resonant tank with series-series (SS) external capacitors acts as a band pass filter, the effect of any harmonic components of $V_{A B}(t)$ can be neglected, except for the fundamental harmonic component. Therefore, the fundamental harmonic of the square wave voltage $V_{A B}^{F}(t)$ is only considered [21]. From Fourier expansion of the square wave voltage $V_{A B}(t)$, the fundamental voltage $V_{A B}^{F}(t)$ is given by

$$
\mathrm{V}_{A B}^{F}(t)=\frac{4}{\pi} V_{I N} \sin \omega t=V_{m} \sin \omega t
$$




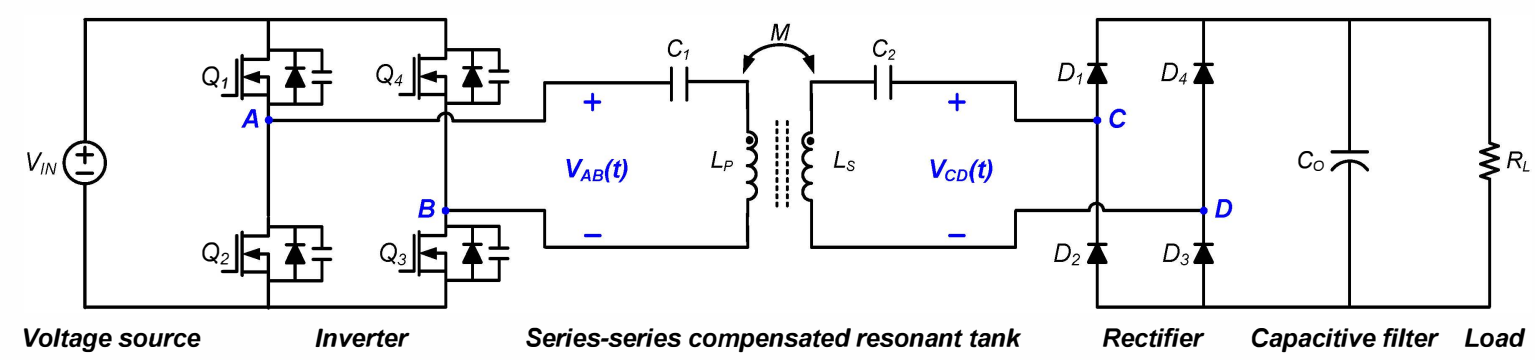

Fig. 1 Configuration of series-series compensated wireless power transfer system

The loosely coupled primary winding and secondary winding is usually modeled by a coupled-inductor model, as shown in Fig. 1. Assuming that the diameter of the primary winding is the same diameter as the secondary winding, the coupled-inductor model is transformed into the two-leakage inductance transformer model, as shown in Fig. 2(a).

For resonance and compensation, external series resonant capacitors $C_{1}$ and $C_{2}$ are added to the two-leakage inductance transformer model. Therefore, the resonant tank of the SSWPT system is formed from two-leakage inductance transformer model and series resonant capacitors. Since the two-leakage inductance transformer model tank with series resonant capacitors has a current-source characteristic, it should be connected to a capacitive filter having a voltagesource characteristic. Therefore, the full-bridge rectifier with the capacitive filter provides DC voltage at the output, which can be represented as a load resistance $R_{L}$. Since the fundamental harmonic of the square wave $V_{A B}^{F}(t)$ at the input is considered, the full-bridge rectifier, capacitive filter, and load can be modeled by ac load resistance $\frac{8}{\pi^{2}} R_{L}$ [21], as shown in Fig. 2(a).

To analyze the resonant tank effectively, the components in the secondary side of the ideal transformer are transformed to their equivalents in the primary side in Fig. 2(b). The ac load resistance is transformed to the equivalent load resistance $R_{\text {eq }}$.

$$
R_{e q}=\frac{8}{\pi^{2}} n^{2} R_{L}
$$

The primary leakage inductance $L_{l k p}$ is equal to the secondary leakage inductance reflected to the primary side $n^{2} L_{l k s}$ in Fig. 2(b).

$$
L_{l k p}=(1-\kappa) L_{P}=(1-\kappa) n^{2} L_{S}=n^{2} L_{l k s}
$$

where $\kappa$ is coupling coefficient.

Also, since the resonant frequency in the primary side is the same as the resonant frequency in the secondary side, the primary resonant capacitance $C_{l}$ is equal to the secondary resonant capacitance reflected to the primary side $C_{2} / n^{2}$, and the reactance $X_{1}$ is equal to the reactance $X_{2}$ in Fig. 2(b).

$$
\begin{aligned}
& C_{1}=\frac{C_{2}}{n^{2}} \quad\left(\frac{1}{\sqrt{L_{P} C_{1}}}=\frac{1}{\sqrt{n^{2} L_{S} C_{1}}}=\frac{1}{\sqrt{L_{S} C_{2}}}\right) \\
& X_{1}=\omega L_{l k p}-\frac{1}{\omega C_{1}}=\omega n^{2} L_{l k s}-\frac{n^{2}}{\omega C_{2}}=X_{2}
\end{aligned}
$$

And then, the reactance in the SS compensated resonant tank is represented as the primary reactance. As a result, the frequency-domain equivalent circuit is shown in Fig. 2(c).

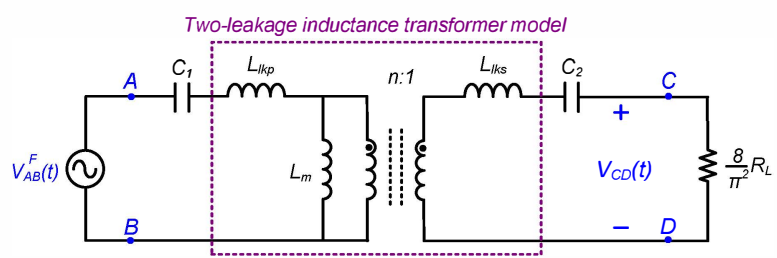

(a)

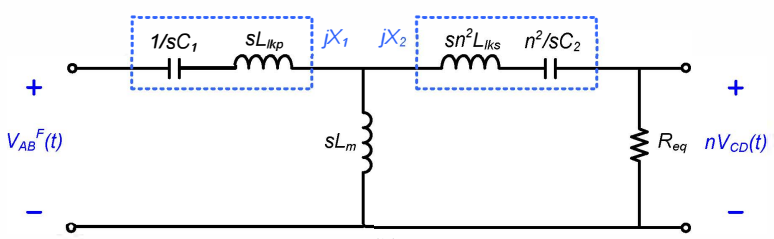

(b)

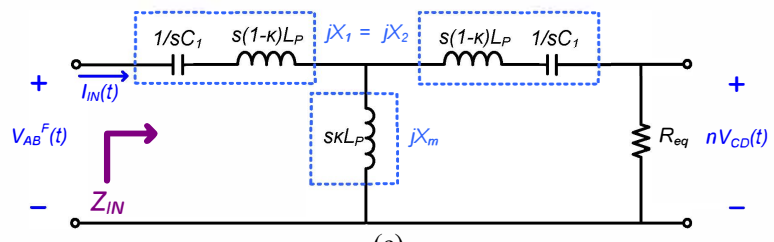

(c)

Fig. 2 Equivalent circuit of series-series compensated wireless power transfer system and its frequency-domain equivalent circuits

\section{RESONANCE FREQUENCIES}

From Fig. 2(c), resonant frequencies are defined from the well-known open-circuit and short-circuit tests. The input impedance under the secondary open-circuit condition $Z_{I N-o c}$ is given by

$$
Z_{I N-o c}=s(1-\kappa) L_{P}+\frac{1}{s C_{1}}+s \kappa L_{P}=\frac{s^{2} L_{P} C_{1}+1}{s C_{1}}
$$

In (7), the input impedance under the secondary open-circuit condition $Z_{I N-o c}$ has two complex conjugate zeros at the first resonant frequency $\omega_{O I}$ and one pole at zero. And then, the input impedance under the secondary short-circuit condition $Z_{I N-s c}$ is given by

$$
\begin{aligned}
Z_{I N-s c} & =s(1-\kappa) L_{P}+\frac{1}{s C_{1}}+s \kappa L_{P} \|\left(s(1-\kappa) L_{P}+\frac{1}{s C_{1}}\right) \\
& =\frac{\left[s^{2}(1-\kappa) L_{P} C_{1}+1\right]\left[s^{2}(1+\kappa) L_{P} C_{1}+1\right]}{s C_{1}\left[s^{2} L_{P} C_{1}+1\right]}
\end{aligned}
$$

In (8), the input impedance has two pair of complex conjugate zeroes at the second and third resonant frequencies $\omega_{O 2}$ and $\omega_{O 3}$, one pole at zero and two complex conjugate zero at the 

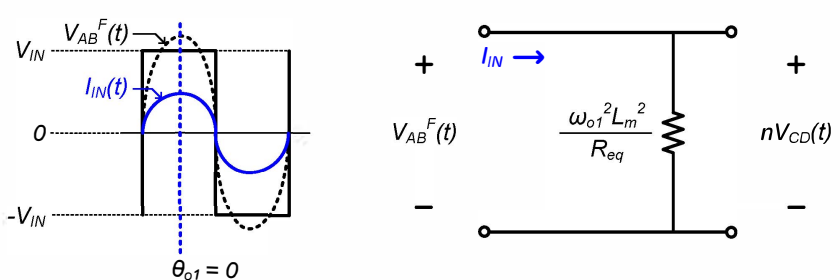

(a)
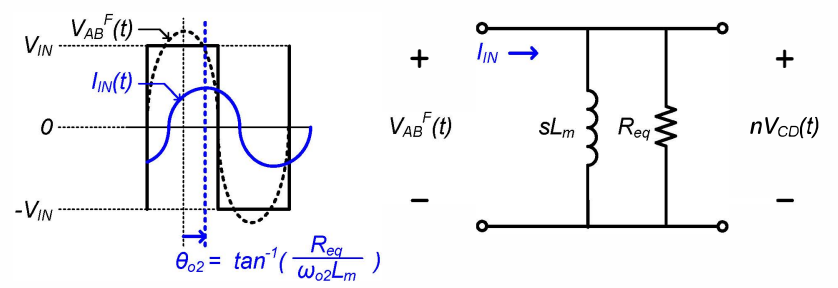

(b)

Fig. 3 Input current and equivalent circuits by input impedance analysis: (a) at first resonant frequency $\omega_{O l}(\mathrm{~b})$ at second resonant frequency $\omega_{O 2}$

first resonant frequency $\omega_{O I}$. Therefore, three resonant frequencies in SS-WPT system are as follows:

$$
\begin{aligned}
& \omega_{O 1}=\frac{1}{\sqrt{L_{P} C_{1}}}=\frac{1}{\sqrt{L_{S} C_{2}}} \\
& \omega_{O 2}=\frac{1}{\sqrt{(1-\kappa) L_{P} C_{1}}}=\frac{1}{\sqrt{L_{l k p} C_{1}}}=\frac{\omega_{O 1}}{\sqrt{1-\kappa}} \\
& \omega_{O 3}=\frac{1}{\sqrt{(1+\kappa) L_{P} C_{1}}}=\frac{1}{\sqrt{\left(L_{P}+L_{m}\right) C_{1}}}=\frac{\omega_{O 1}}{\sqrt{1+\kappa}}
\end{aligned}
$$

The first resonant frequency $\omega_{O I}$ is fixed. On the other hand, the second and third resonant frequencies $\omega_{O 2}$ and $\omega_{O 3}$ are determined by the coupling coefficient $\kappa$.

The second resonant frequency $\omega_{\mathrm{O} 2}$ is preferred in Power Electronics, whereas the first resonant frequency $\omega_{O 1}$ is preferred in other research areas. Therefore, the first and second resonant frequency $\omega_{\mathrm{O} 1}$ and $\omega_{\mathrm{O} 2}$ are mainly analyzed in this paper.

\section{INPUT IMPEDANCE ANALYSIS}

With the known input voltage $V_{A B}^{F}(t)$, the input impedance $Z_{I N}$ provides the information of input current $I_{I N}(t)$, which determines the operation of inverter from the phase difference between voltage and current at the input. The input impedance $Z_{I N}$ of the SS-WPT system in Fig. 2(c) can be obtained as:

$$
Z_{I N}=j X_{1}+j X_{m} \|\left(j X_{2}+R_{e q}\right)=j X_{1}+\frac{j X_{m}\left(j X_{1}+R_{e q}\right)}{j X_{m}+j X_{1}+R_{e q}}
$$

The input impedance $Z_{I N}$ is investigated at the two major resonant frequencies $\omega_{\mathrm{O}}$ and $\omega_{\mathrm{O} 2}$ to analyze the SS-WPT system at the resonant frequencies.

At the first resonant frequency $\omega_{O l}$, the reactance $X_{1}$ and the reactance $X_{m}$ can be written as

$$
\begin{aligned}
& X_{1}=\omega L_{l k p}-\frac{1}{\omega C_{1}}=\omega L_{P}\left(1-\frac{\omega_{O 1}^{2}}{\omega^{2}}-\kappa\right)=-\omega_{O 1} \kappa L_{P} \\
& X_{m}=\omega_{O 1} \kappa L_{P}
\end{aligned}
$$

At the first resonant frequency $\omega_{O I}$, the input impedance $Z_{I N}$ and the phase difference $\theta_{O I}$ can be written by

$$
\begin{aligned}
& Z_{I N\left(\omega=\omega_{O 1}\right)}=-\mathrm{j} \omega_{O 1} \kappa L_{P}+\frac{\mathrm{j} \omega_{O 1} \kappa L_{P}\left(-\mathrm{j} \omega_{O 1} \kappa L_{P}+R_{e q}\right)}{R_{e q}} \\
& =\frac{\omega_{O 1}{ }^{2} k^{2} L_{P}{ }^{2}}{R_{e q}}=\frac{\omega_{O 1}{ }^{2} L_{m}{ }^{2}}{R_{e q}}=\kappa^{2} Q_{L}{ }^{2} R_{e q} \\
& \theta_{O 1}=\tan ^{-1}(0)=0 \\
& Q_{L}=\frac{\omega_{O 1} L_{P}}{R_{e q}}
\end{aligned}
$$

where $Q_{L}$ is load quality factor

Since the input impedance $Z_{I N}$ contains no imaginary term in (15), the input impedance is resistive and the equivalent circuit at the first resonant frequency $\omega_{O I}$ consists of only a resister, as shown in Fig. 3(a). Thus, the input current $I_{I N}(t)$ is in phase with the input voltage $V_{A B}^{F}(t)$, as shown in Fig. 3(a). As a result, all switches in the inverter are turned on and off at zero current. Also, the equivalent load resistance $R_{e q}$ is in inverse proportion to the input impedance $Z_{I N}$. Thus, the input current $I_{I N}(t)$ increases at the lighter load due to the low input impedance. That is, SS-WPT system has poor output voltage regulation at the first resonant frequency $\omega_{O 1}$.

At the second resonant frequency $\omega_{O 2}$, the reactance $X_{I}$ and $X_{m}$ can also be written as

$$
\begin{aligned}
& X_{1}=\omega L_{l k p}-\frac{1}{\omega C_{1}}=\omega L_{P}\left(1-\frac{\omega_{O 2}^{2}}{\omega^{2}}\right)=0 \\
& X_{m}=\omega_{O 2} \kappa L_{P}
\end{aligned}
$$

The input impedance $Z_{I N}$ and the phase difference $\theta_{O 2}$ at the second resonant frequency $\omega_{O 2}$ can be written by

$$
\begin{aligned}
& Z_{I N\left(\omega=\omega_{O 2}\right)}=\frac{j \omega_{o 2} \kappa L_{P} R_{e q}}{j \omega_{o 2} k L_{P}+R_{e q}}=\frac{j \omega_{o 2} L_{m} R_{e q}}{j \omega_{o 2} L_{m}+R_{e q}}=s L_{m} \| R_{e q} \\
& \theta_{O 2}=\tan ^{-1}\left(\frac{R_{e q}}{\omega_{O 2} \kappa L_{P}}\right)=\tan ^{-1}\left(\frac{R_{e q}}{\omega_{o 2} L_{m}}\right)
\end{aligned}
$$

The equivalent circuit at the second resonant frequency $\omega_{O 2}$ consists of the equivalent load resistance $R_{e q}$ and the magnetizing inductance $L_{m}$ from (20), as shown in Fig. 3(b). As a result, the input impedance is inductive and the input current $I_{I N}(t)$ lags the input voltage $V_{A B}^{F}(t)$ by the phase difference $\theta_{O 2}$ in (21), as shown in Fig. 3(b). Thus, all switches in the inverter can be turned on with zero-voltage-switching (ZVS). Also, unlike the input impedance at the first resonant frequencies $\omega_{O 1}$, the input current $I_{I N}(t)$ decreases at the lighter load

Note that the input impedances $Z_{I N}$ at the second resonant frequencies $\omega_{\mathrm{O}_{2}}$ in (20) are about the same with the equivalent load resistance $R_{e q}$ and the phase differences $\theta_{O 2}$ in (21) become almost zero when the terms $\omega_{O_{2}} \kappa L_{P}$ is much more than the equivalent load resistance $R_{e q}$ (the load quality factor $Q_{L}$ is very high).

$$
\begin{gathered}
Z_{I N\left(\omega=\omega_{O 2}\right)} \approx R_{e q} \\
\theta_{O 2} \approx 0
\end{gathered}
$$

\section{FHA-BASED FREQUENCY ANALYSIS}

The FHA-based frequency analysis is carried out to help more understand the operation of the SS-WPT system graphically. The FHA-based frequency analysis consists of voltage gain $G_{V}$, current gain $G_{I}$, and power gain $G_{P}$. 


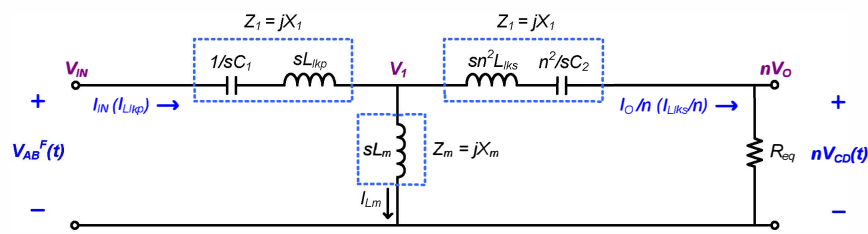

Fig. 4 Equivalent circuit for fundamental harmonic approximation-based frequency analysis

\section{A. Voltage Gain $G_{V}$}

From Fig. 4, the voltage gain $G_{V}$ is as follows:

$$
\left.G_{V}=\left\{\left[\frac{1}{\kappa}\left(1-\frac{1}{\omega_{n}^{2}}\right)\right]^{2}+\left[Q_{L} \omega_{n}\left(\frac{1}{\kappa}\left(1-\frac{1}{\omega_{n}^{2}}\right)^{2}-\kappa\right)\right)\right]^{2}\right\}^{-0.5}
$$

where the normalized frequency $\omega_{n}$ is defined by

$$
\omega_{n}=\omega / \omega_{O 1}
$$

The voltage gains $G_{V}$ at the first and second resonant frequency $\omega_{O 1}$ and $\omega_{O 2}$ can be obtained as:

$$
\begin{aligned}
& G_{V\left(\omega=\omega_{O 1}\right)}=\left|\frac{R_{e q}}{-\mathrm{j} \kappa \omega_{O_{1}} L_{P}}\right|=\frac{R_{e q}}{\omega_{O 1} L_{m}}=\frac{R_{e q}}{\kappa Z_{O 1}}=\frac{1}{\kappa Q_{L}} \\
& G_{V\left(\omega=\omega_{O 2}\right)}=\left|\frac{\mathrm{j} \kappa \omega_{O 2} L_{P} R_{e q}}{\mathrm{j} \kappa \omega_{O 2} L_{P} R_{e q}}\right|=\left|\frac{\mathrm{j} \omega_{O 2} L_{m} R_{e q}}{\mathrm{j} \omega_{O 2} L_{m} R_{e q}}\right|=1
\end{aligned}
$$

Fig. 5 shows the voltage gain $G_{V}$ of the SS-WPT system according to three key parameters of loaded quality factor $Q_{L}$ : first resonant frequency $f_{O l}$, self-inductance $L_{P}$, and load resistance $R_{L}$. The voltage gain $G_{V}$ at the first resonant frequency $\omega_{O I}$ decreases as the load quality factor $Q_{L}$ increases, as shown in Fig. 5. Since the equivalent load resistance $R_{e q}$ is in inverse proportion to the input impedance $Z_{I N}$. SS-WPT system has very poor output voltage regulation at the first resonant frequency $\omega_{O I}$. On the other hand, as long as the inverter operates at the second resonant frequencies $\omega_{O_{2}}$ (or at the third resonant frequencies $\omega_{O 3}$ ), the voltage gain $G_{V}$ is always one since the reactance $X_{1}$ is zero at the second resonant frequencies $\omega_{\mathrm{O} 2}$.

When the voltage gain $G_{V}$ at the first resonant frequency is much higher than one due to the low load quality factor $Q_{L}$, the voltage gain $G_{V}$ has a single peak. And then, as the load quality factor $Q_{L}$ increases, the voltage gain $G_{V}$ decreases and becomes almost one. At that time, a single peak is being divided into a double peak. At the moment it is called critical coupling in the view point of Coupled Mode Theory. After that, as the voltage gain $G_{V}$ at the first resonant frequency $\omega_{O I}$ becomes much lower, the distance between two peaks increases.

Fig. 6 shows voltage gain $G_{V}$ of SS-WPT system according to coupling coefficient $\kappa$. The first resonant frequency $\omega_{O I}$ is fixed and the voltage gain $G_{V}$ at the first resonant frequency $\omega_{O I}$ decreases, as the coupling coefficient $\kappa$ increases. On the other hand, the second resonant frequencies $\omega_{O 2}$ is variable although the voltage gain $G_{V}$ at the second resonant frequencies $\omega_{\mathrm{O}_{2}}$ is always one.

\section{B. Current Gain $G_{I}$ and Power Gain $G_{P}$}

In a similar way to the voltage gain $G_{V}$, the current gain $G_{I}$ is as follows:

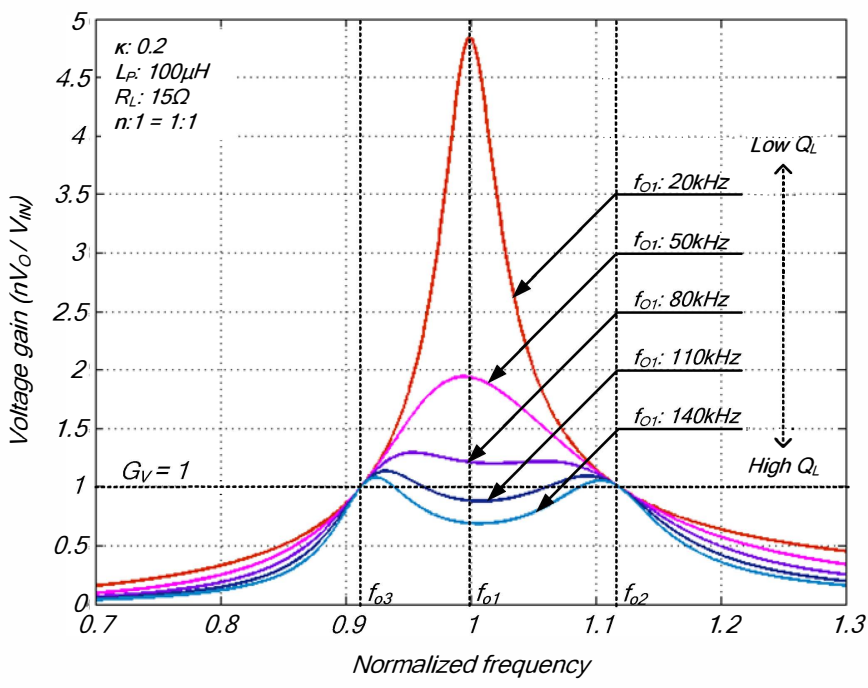

(a)

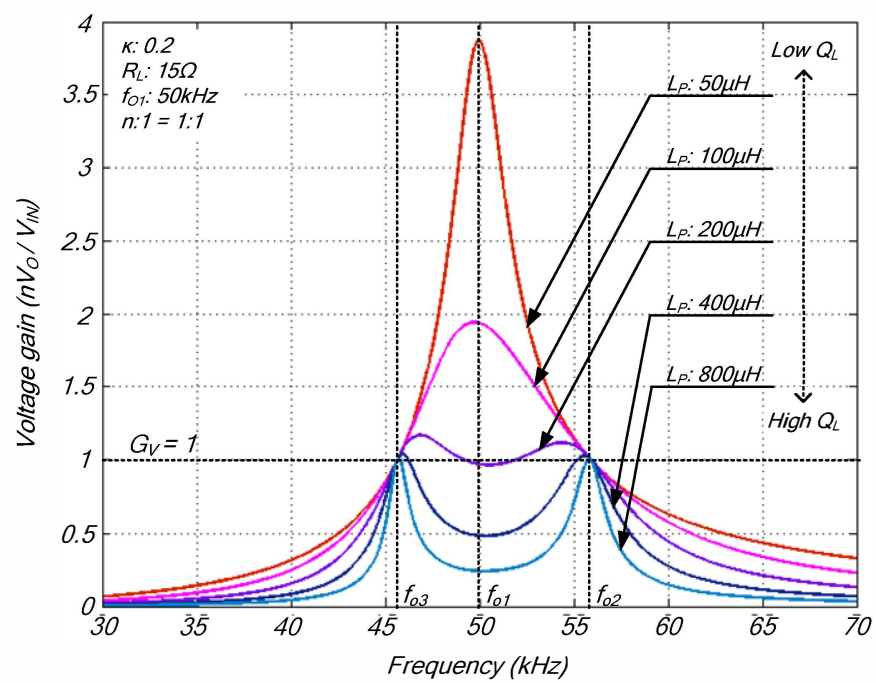

(b)

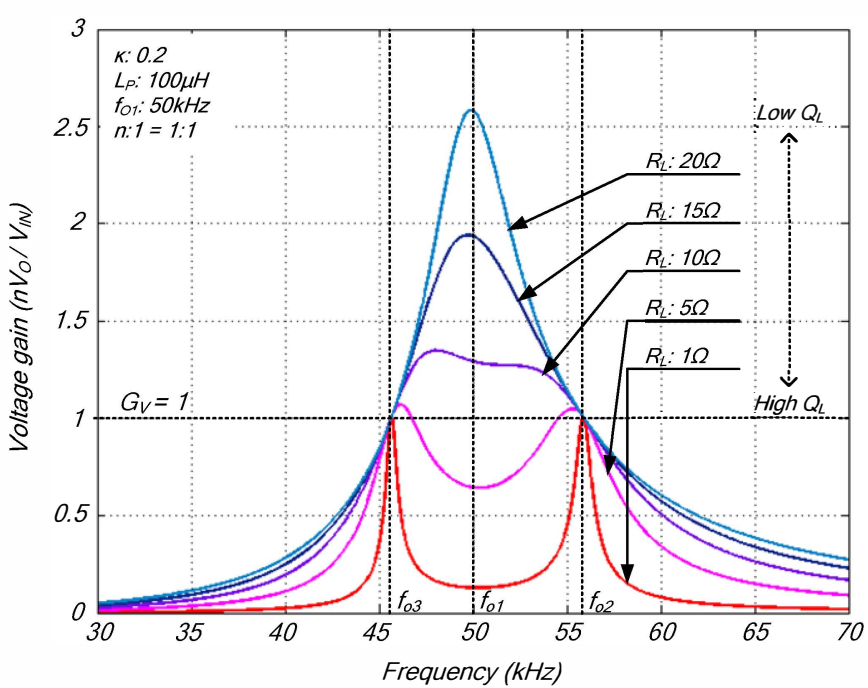

(c)

Fig. 5 Voltage gain $G_{V}$ according to loaded quality factor (a) first resonant frequency $f_{O I}(\mathrm{~b})$ self-inductance $L_{P}(\mathrm{c})$ load resistance $R_{L}\left(f_{O l}=50 \mathrm{kHz}, n: 1=\right.$ $1: 1)$ 


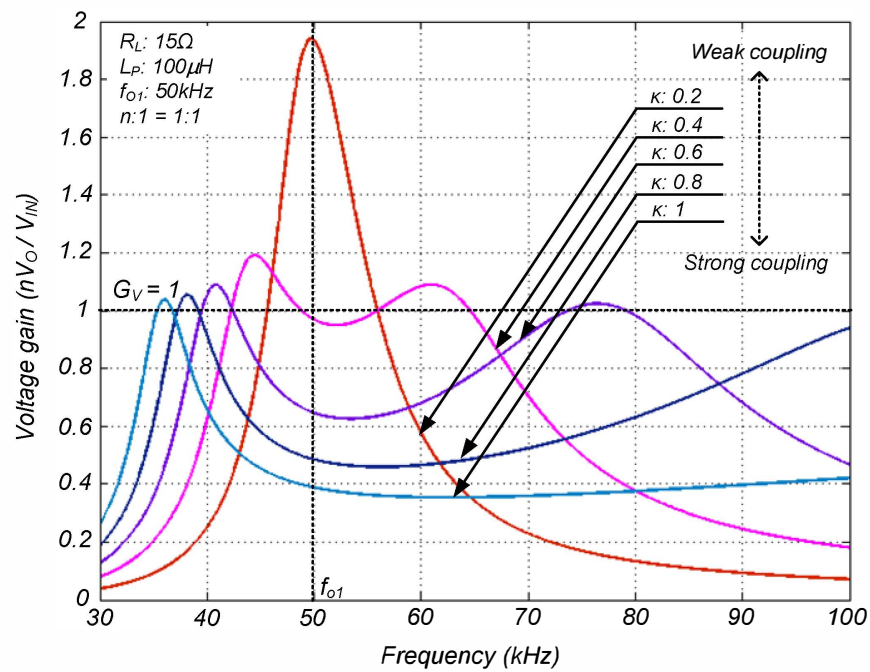

Fig. 6 Voltage gain $G_{V}$ according to coupling coefficient $\kappa\left(R_{L}=15 \Omega, L_{P}=\right.$ $\left.100 \mu \mathrm{H}, f_{O l}=50 \mathrm{kHz}, n: l=1: 1\right)$

$$
G_{I}=\left\{\left[\frac{1}{\kappa}\left(1-\frac{1}{\omega_{n}^{2}}\right)\right]^{2}+\left[\frac{1}{\kappa Q_{L} \omega_{n}}\right]^{2}\right\}^{-0.5}
$$

The current gains $G_{I}$ at the first and second resonant frequency $\omega_{O 1}$ and $\omega_{O 2}$ can be obtained as:

$$
\begin{aligned}
G_{I\left(\omega=\omega_{O 1}\right)} & =\left|\frac{j \kappa \omega_{O 1} L_{P}}{R_{e q}}\right|=\kappa Q_{L} \\
G_{I\left(\omega=\omega_{O 2}\right)} & =\left|\frac{j \kappa \omega_{O 2} L_{P}}{R_{e q}+j \kappa \omega_{O 2} L_{P}}\right|=\left|\frac{j \omega_{O 2} L_{m}}{R_{e q}+j \omega_{O 2} L_{m}}\right|
\end{aligned}
$$

From the above-mentioned the voltage gain $G_{V}$ and the current gain $G_{I}$, the power gain $G_{P}$ can be obtained as:

$$
G_{P}=G_{V} G_{I}
$$

The power gains $G_{P}$ at the first and second resonant frequency $\omega_{O 1}$ and $\omega_{O 2}$ can be obtained as:

$$
\begin{aligned}
& G_{P\left(\omega=\omega_{01}\right)}=\frac{1}{\kappa Q_{L}} \kappa Q_{L}=1 \\
& G_{P\left(\omega=\omega_{O 2}\right)}=\left|\frac{j \kappa \omega_{O 2} L_{P}}{R_{e q}+j \kappa \omega_{O_{2}} L_{P}}\right|=\left|\frac{j \frac{\kappa \omega_{O 2} L_{P}}{R_{e q}}}{1+j \frac{\kappa \omega_{O 2} L_{P}}{R_{e q}}}\right|=\left|\frac{j \frac{\kappa}{\sqrt{1-\kappa}} Q_{L}}{1+j \frac{\kappa}{\sqrt{1-\kappa}} Q_{L}}\right|
\end{aligned}
$$

Fig. 7 shows the characteristics of the power gain $G_{P}$ versus frequency according to the load quality factor $Q_{L}$ : first resonant frequency $f_{O l}$, self-inductance $L_{P}$, and load resistance $R_{L}$. As long as the inverter operates at the first resonant frequency $\omega_{O l}$, the power gain $G_{P}$ keeps unity. On the other hand, the power gains $G_{P}$ at the second resonant frequency $\omega_{\mathrm{O} 2}$ is less than one. However, when load quality factor $Q_{L}$ is very high, the power gains $G_{P}$ at the second resonant frequency $\omega_{O 2}$ increases to almost unity.

At the very low load quality factor $Q_{L}$, the power gain has a single sharp peak. And then, as the load quality factor $Q_{L}$ increases, the single sharp peak is changed into a single blunt peak. After that, a single peak is divided into a trident peak as the load quality factor $Q_{L}$ becomes much higher. At the very high load quality factor $Q_{L}$, the trident peak becomes much sharper.

Also, Fig. 9 shows the characteristics of the power gain $G_{P}$ versus frequency according to the coupling coefficient $\kappa$.

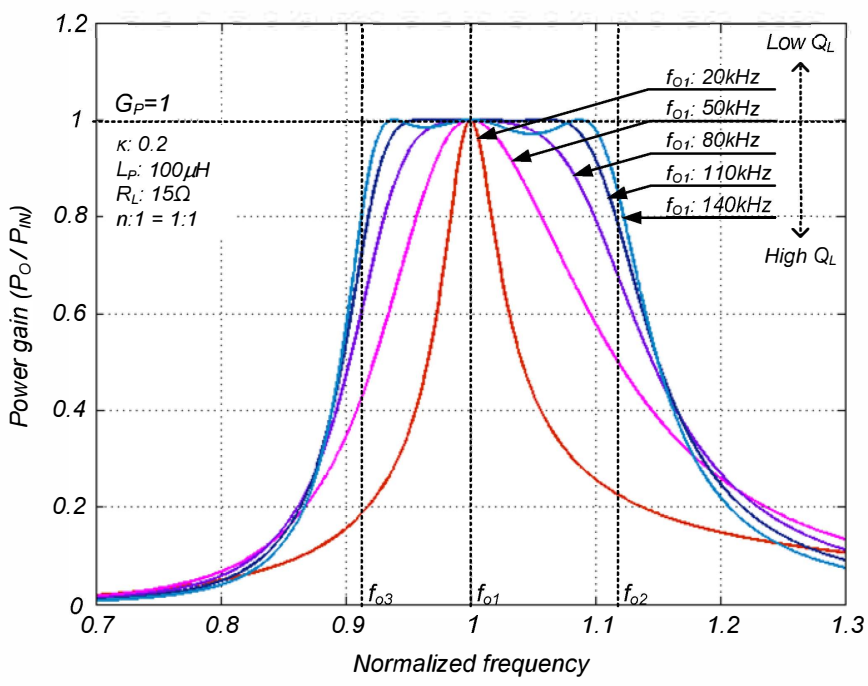

(a)

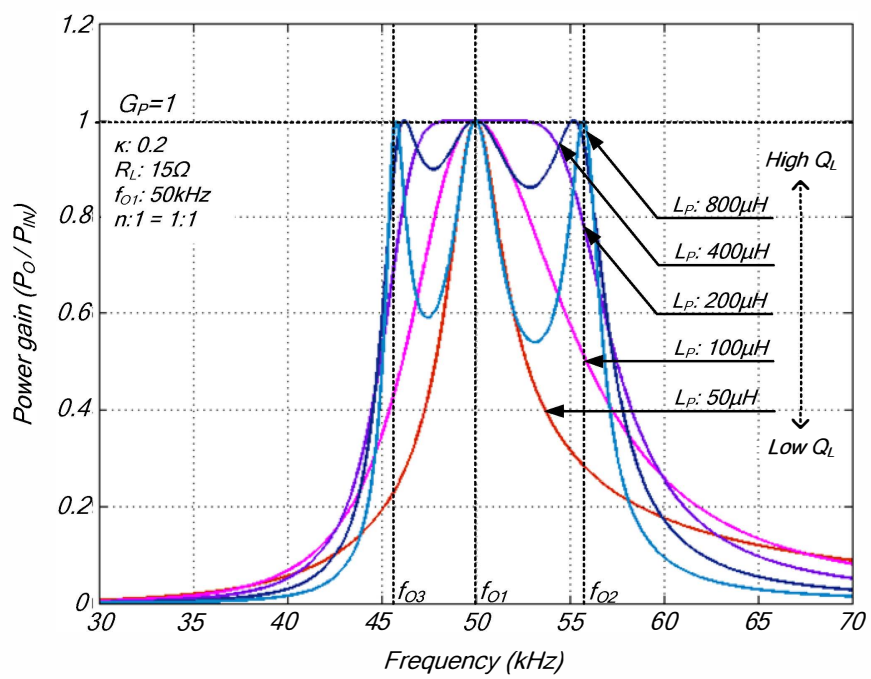

(b)

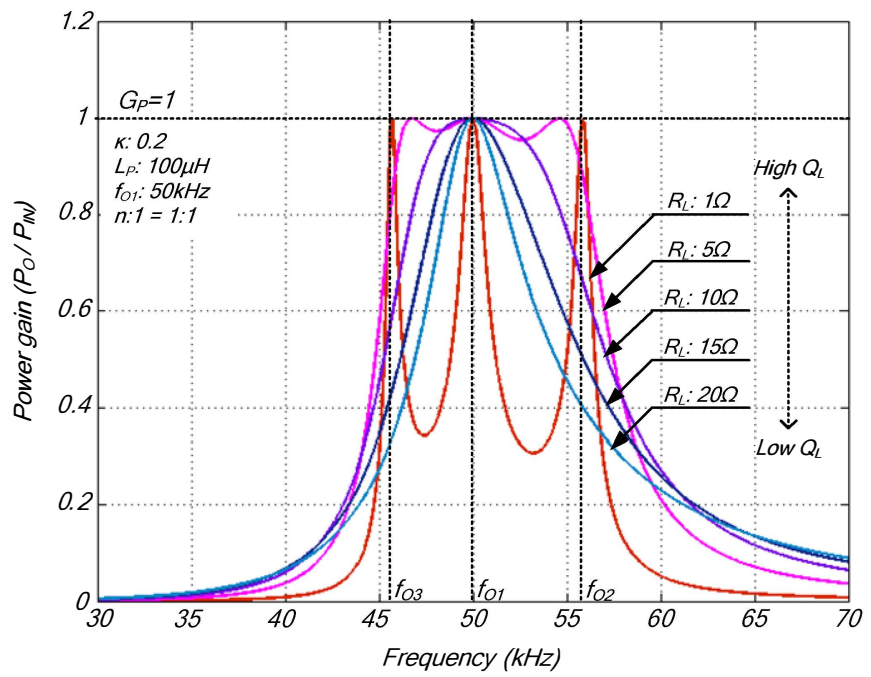

(c)

Fig. 7 Power gain $G_{V}$ according to loaded quality factor (a) first resonant frequency $f_{O I}(\mathrm{~b})$ self-inductance $L_{P}(\mathrm{c})$ load resistance $R_{L}\left(f_{O I}=50 \mathrm{kHz}, n: 1=\right.$ $1: 1)$ 


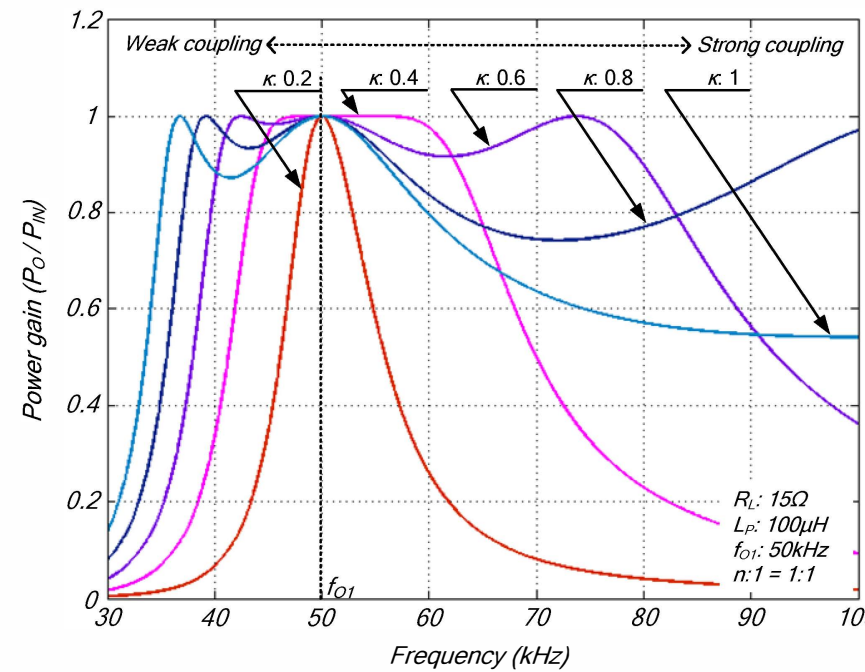

Fig. 8 Power gain $G_{V}$ according to coupling coefficient $\kappa\left(R_{L}=15 \Omega, L_{P}=\right.$ $\left.100 \mu \mathrm{H}, f_{O l}=50 \mathrm{kHz}, n: l=1: 1\right)$
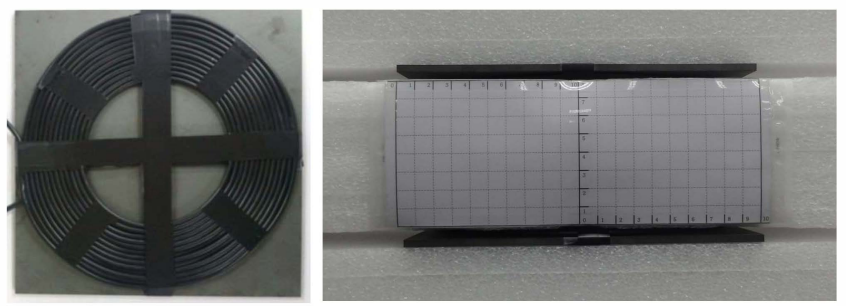

Fig. 9 Resonator (size: $20 \mathrm{~cm} \times 20 \mathrm{~cm}$, separated distance: $8 \mathrm{~cm}$ and equivalent AWG: 14)

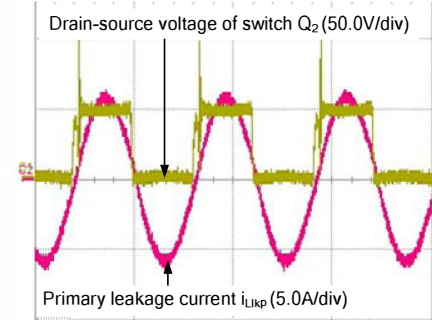

(a)

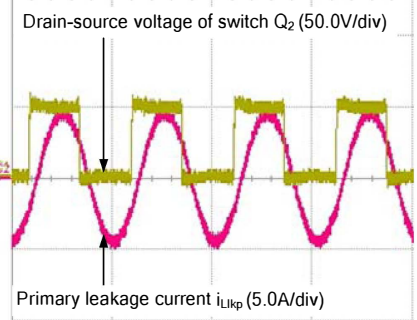

(b)
Fig. 10 Experimental waveforms (a) at the first resonant frequency $\omega_{\mathrm{O} 1}(\mathrm{~b})$ at the second resonant frequency $\omega_{\mathrm{O} 2}$ (Time: $5 \mu \mathrm{s} / \mathrm{div}, V_{I N}=50 \mathrm{~V}$ and $\mathrm{R}_{\mathrm{L}}: 15.5 \Omega$ )

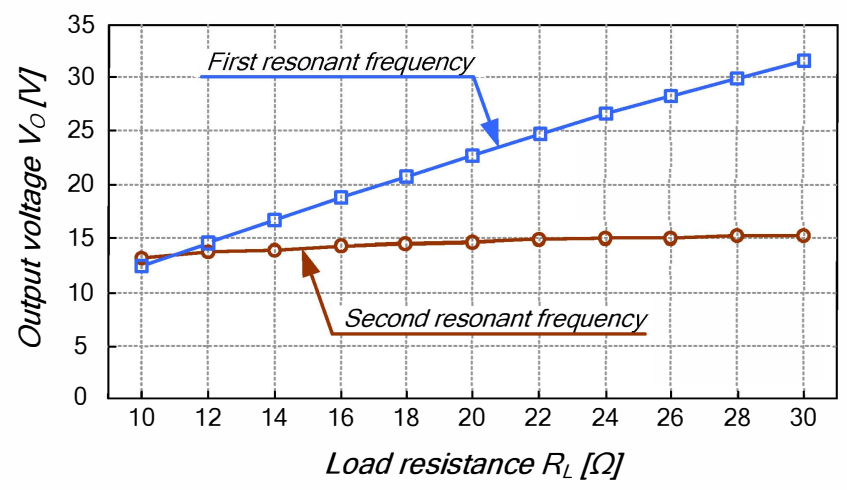

Fig. 11 Output voltage according to load resistance $R_{L}$ at the first resonant frequency $\omega_{O I}$ and at the second resonant frequency $\omega_{O 2}\left(V_{I N}=20 \mathrm{~V}\right)$

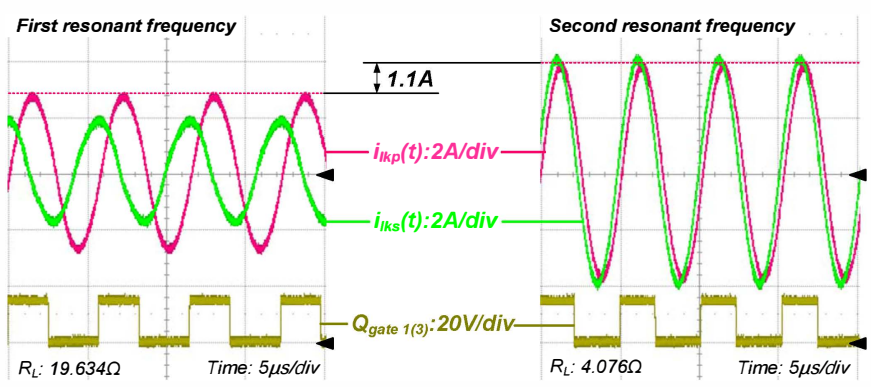

(a)

(b)

Fig. 12 Experimental waveform at the same output power (a) at the first resonant frequency $\omega_{O I}(\mathrm{~b})$ at the second resonant frequency $\omega_{O 2}($ Time: $5 \mu \mathrm{s} / \mathrm{div}$ and $V_{I N}=20 \mathrm{~V}$ )

Regardless of the variation of the coupling coefficient $\kappa$, the power gain $G_{P}$ keeps unity. Theoretically, at the first resonant frequency $\omega_{O I}$, the input power $P_{I N}$ is totally transferred to the output since the input impedance $Z_{I N}$ contains no imaginary term and the impedance matching is perfectly accomplished at the resonant frequency $\omega_{O I}$. Therefore, low coupling coefficient $\kappa$ by long separated distance does not matter at the first resonant frequency $\omega_{O I}$.

On the other hand, at the second resonant frequency $\omega_{\mathrm{O} 2}$, the power gain $G_{P}$ is less than one from (31) when the load quality factor $Q_{L}$ and the coupling coefficient $\kappa$ are low. That is, a part of input power $P_{I N}$ is transferred to the output because of the low current gain $G_{I}$. However, when the load quality factor $Q_{L}$ and the coupling coefficient $\kappa$ are very high, the low current gain $G_{I}$ and the power gain $G_{P}$ is almost one at the second resonant frequency $\omega_{O 2}$. In other words, when the magnetic coupling is loose and the coupling coefficient $\kappa$ is low, the very high load quality factor $Q_{L}$ is required for high efficiency. Thus, when the load quality factor $Q_{L}$ is not high, the separated distance is limited at the second resonant frequency $\omega_{O 2}$.

\section{EXPERIMENTAL VERIFICATION}

To support the analysis, experimental results are performed with specifications of the self-inductance $L_{P}=L_{S}=55.9 \mu \mathrm{H}$ (Fig. 9), the resonant capacitor $C_{l}=C_{2}=15 \mathrm{nF}$, the separation distance $8 \mathrm{~cm}$, and coupling coefficient $\kappa=0.19$.

Fig. 10 shows the phase difference between the voltage and current at the input. Since the input impedance at the first resonant frequency $\omega_{O I}$ is resistive, the voltage waveform and current waveform are in phase, as shown in Fig. 10(a). On the other hand, since the input impedance at the second resonant frequency $\omega_{O 2}$ is inductive, the current waveform lags the voltage waveform, as shown in Fig. 10(b). At that time, all the MOSFET are turned on with ZVS.

Fig. 11 shows the output voltage $V_{O}$ according to load resistance $R_{L}$ at the first resonant frequency $\omega_{O I}$ and at the second resonant frequency $\omega_{O 2}$. At the first resonant frequency $\omega_{O I}$, the input impedance $Z_{I N}$ is in reverse proportion to the equivalent load resistance $R_{\text {eq }}$. For this reason, as the load resistance $R_{L}$ increases, the input impedance $Z_{I N}$ decreases. And then, since the input current and input power increase, the output voltage $V_{O}$ also increases. On the other hand, since the output voltage at the second resonant frequency $\omega_{O_{2}}$ is insensitive to load resistance $R_{L}$, the output voltage $V_{O}$ is almost constant. 
Also, when the coupling coefficient $\kappa$ are very low, the power gain $G_{P}$ at the first resonant frequency $\omega_{O I}$ is higher than that at the second resonant frequency $\omega_{\mathrm{O} 2}$. Therefore, the higher input current (primary current) at the second resonant frequency $\omega_{\mathrm{O} 2}$ is required to transfer the same output voltage to a load, as shown in Fig. 12.

\section{CONCLUSION}

To understand WPT system more easily, this paper presents the features of the SS-WPT system in two major resonant frequencies: one resonant frequency by self-inductance and resonant capacitor and the other resonant frequency by leakage inductance and resonant capacitor.

From the input impedance analysis, this paper shows that the phase difference at two resonant frequencies determines the operation of the inverter. Also, from the voltage gain $G_{V}$ in the FHA-based frequency analysis, this paper describes that the operation at the second resonant frequencies $\omega_{\mathrm{O} 2}$ is suitable for a wide range of load variation due to the characteristic of good load regulation. Furthermore, from the power gain $G_{P}$ in the FHA-based frequency analysis, this paper explains that the operation at the first resonant frequency $\omega_{O I}$ is preferred for transferring power in large separation distance.

\section{REFERENCES}

[1] A. Karalis, J. Joannopoulos, and M. Soljacic, "Efficient wireless nonradiative mid-range energy transfer," Ann. Phys., vol. 323, no. 1, pp. 3448, 2008, January Special Issue 2008

[2] A. Kurs, A. Karalis, R. Moffatt, J. D. Joannopoulos, P. Fisher, and M. Soljacic, "Wireless power transfer via strongly coupled magnetic resonances," Science, vol. 317, no. 5834, pp. 83-86, Jul. 2007.

[3] A. Kurs, R. Moffatt and M. Soljacic "Simultaneous mid-range power transfer to multiple devices", Appl. Phys. Lett., vol. 96, no. 4, pp.044 102, Jan. 2010

[4] R. E. Hamam , A. Karalis , J. D. Joannopoulos and M. Soljacic "Efficient weakly-radiative wireless energy transfer: An EIT-like approach", Ann. Phys., vol. 324, no. 8, pp.1783 -1795 2009

[5] N. Yin, G. Xu, Q. Yang, J. Zhao, X. Yang, J. Jin, W. Fu, and M. Sun , "Analysis of Wireless Energy Transmission for Implantable Device Based on Coupled Magnetic Resonance" IEEE Tanns. Magnetics, vol. 48, no.2, Feb 2012

[6] S. Hackworth, X. Liu, C. Li, and M. Sun "Wireless solar energy to homes: A magnetic resonance approach", International Journal of Innovations in Energy Systems and Power, vol. 5, no.1, April 2010

[7] F. Zhang, S. A. Hackworth, X. Liu, C. Li and M. Sun, "Wireless Power Delivery for Wearable Sensors and Implants in Body Sensor Networks," 32nd Annual International Conference of the IEEE Engineering in Medicine and Biology Society, pp. 692-695, Argentina, 31 August-4 Sept 2010
[8] F. Zhang, J. Liu, Z. Mao and M. Sun, "Mid-Range Wireless Power Transfer and Its Application to Body Sensor Networks," Open Journal of Applied Sciences, Vol. 2 No. 1, pp. 35-46, 2012

[9] A. P. Sample, et al., "Analysis, Experimental Results, and Range Adaptation of Magnetically Coupled Resonators for Wireless Power Transfer," IEEE Transactions on Industrial Electronics, vol. 58, pp. 544-554, Feb 2011

[10] T. Imura, "Study on maximum air-gap and efficiency of Magnetic Resonant Coupling for Wireless Power Transfer using Equivalent Circuit," Industrial Electronics (ISIE), 2010 IEEE International Symposium on, pp. 3664-3669, 2010.

[11] Sanghoon Cheon, Yong-Hae Kim, Seung-Youl Kang, Myung Lae Lee, Jong-Moo Lee, Taehyoung Zyung, "Circuit-Model-Based Analysis of a Wireless Energy-Transfer System via Coupled Magnetic Resonances", Industrial Electronics, IEEE Transactions on, vol. 58, no. 7, pp: $2906-$ 2914, July 2011

[12] T. Imura and Y. Hori, "Maximizing Air Gap and Efficiency of Magnetic Resonant Coupling for Wireless Power Transfer Using Equivalent Circuit and Neumann Formula," IEEE Transactions on Industrial Electronics, vol. 58, pp. 4746-4752, Oct 2011

[13] Y. H. Kim, et al., "Optimization of Wireless Power Transmission through Resonant Coupling," Cpe: 2009 Compatibility and Power Electronics, pp. 426-431, 2009.G. Eason, B. Noble, and I.N. Sneddon, "On certain integrals of Lipschitz-Hankel type involving products of Bessel functions," Phil. Trans. Roy. Soc. London, vol. A247, pp. 529551, April 1955. (references)

[14] M. Kiani, U.-M. Jow, and M. Ghovanloo, "Design and optimization of a 3-coil inductive link for efficient wireless power transmission," IEEE Trans. Biomed. Circuits and Syst., vol. 5, no. 6, pp. 579 - 591, Dec. 2011

[15] J. Kim, H.-C. Son, K.-H. Kim, and Y.-J. Park, "Efficiency analysis of magnetic resonance wireless power transfer with intermediate resonant coil," IEEE Antennas and Wireless Propagat. Lett., vol.10, pp.389-392, May. 2011

[16] M. Kiani, and M. Ghovanloo, "The circuit theory behind coupled-mode magnetic resonance-based wireless power transmission," IEEE Trans. Circuits and Systems I., vol. 59, no. 8, Aug. 2012

[17] A. K. RamRakhyani, S. Mirabbasi, and M. Chiao, "Design and optimization of resonance-based efficient wireless power delivery systems for biomedical implants," IEEE Trans. Biomed. Circuits Syst., vol. 5, no. 1, pp. 48-63, Feb. 2011

[18] R. R. Harrison, "Designing efficient inductive power links for implantable devices," in Proc. IEEE Int. Symp. Circuits Syst., pp. 20802083, May 2007.

[19] A.Moradewicz and M. Kazmierkowski, "Contactless Energy Transfer System With FPGA-Controlled Resonant Converter," IEEE Trans. Industrial Electronics., vol. 57, no. 9, pp. 3181-3190,Sep. 2010.

[20] G. Chen, S. Wong, C. Tse and X. Ruan, "Analysis, Design, and Control of a Transcutaneous Power Regulator for Artificial Hearts," IEEE Trans. Biomed. Circuits Syst., vol. 3, no. 1, pp. 23-31, Feb. 2009.

[21] L.Steigerwald, "A comparison of Half-Bridge Resonant Converters," IEEE Trans. Power Electron, Vol.3, No.2, April 1988 